\title{
Actin capping protein regulates actomyosin contractility to maintain germline architecture in $C$. elegans
}

\author{
Shinjini Ray ${ }^{1,2}$, Priti Agarwal ${ }^{1}$, Ronen Zaidel-Bar ${ }^{*}$ \\ ${ }^{1}$ Department of Cell and Developmental Biology, Faculty of Medicine, Tel Aviv University, 6997801, Israel \\ ${ }^{2}$ Graduate program, Mechanobiology Institute, National University of Singapore, 117411, Singapore \\ *Correspondence: zaidelbar@tauex.tau.ac.il
}

\begin{abstract}
Actin dynamics play an important role in the morphogenesis of cells and tissues, yet the control of actin filament growth takes place at the molecular level. A challenge in the field is to link the molecular function of actin regulators with their physiological function. Here, we report the in vivo role of the actin capping protein CAP-1 in the C. elegans germline. We show that CAP-1 is associated with actomyosin structures in the cortex and rachis, where it keeps the level of contractility in check. A $60 \%$ reduction in the level of CAP-1 leads to a 2-fold increase in F-actin and non-muscle myosin II and only a 30\% increase in Arp2/3. CAP-1 depletion leads to severe structural defects in the syncytial germline and oocytes, which can be rescued by reducing myosin activity. Thus, we uncover a physiological role for actin capping protein in maintaining C. elegans fertility by regulating the level of actomyosin contractility.
\end{abstract}

Keywords: actin capping protein, actin dynamics, cytoskeleton, actomyosin, contractility, Caenorhabditis elegans, syncytial germline, formins, Arp2/3 complex, rachis, morphogenesis 


\section{Introduction}

Diverse actin structures support the shape and power the movement of biological entities from the subcellular to the tissue level. Their functional diversity, manifested by different actin network architectures and dynamics, is regulated by their association with a variety of actin binding proteins in a context-dependent manner (Pollard, 2016; Winder and Ayscough, 2005). The barbed end of the actin filament is a hotspot for regulation, where interactions between multiple proteins determines whether it will elongate or remain stable (Shekhar et al., 2016). Capping protein (CP, also known as CAPZ and $\beta$-actinin) is one of the major determinants of barbed end dynamics (Edwards et al., 2014). CP binding at the barbed end of the actin filament prevents addition or loss of actin subunits. It functions as a heterodimer composed of two structurally similar $\alpha$ and $\beta$ subunits (Yamashita et al., 2003).

Cooperative and competitive interactions between $\mathrm{CP}$, nucleators and elongation factors influence the actin network architecture, dynamics and mechanical properties. CP competes with formin, a nucleation and elongation factor, for the barbed end, where they transiently form a ternary 'decision complex'. Binding of formin to a CP-bound barbed end weakens the association of CP and promotes elongation of the filament (Bombardier et al., 2015; Kim et al., 2010; Shekhar et al., 2015). Similarly, CP is in direct competition with the Ena/VASP family of actin elongation factors (Bear and Gertler, 2009). In a branched actin network, nucleated by the Arp $2 / 3$ complex, CP determines the length and thus indirectly controls the density of branching (Funk et al., 2021; Iwasa and Mullins, 2007; Pollard and Cooper, 2009). While Arp2/3 nucleates branches along the actin filaments, CP caps them. Shorter branches provide strength but have less flexibility (Akin and Mullins, 2008; Schaub et al., 2007; Vinzenz et al., 2012). Most of the studies revealing the function of CP at the molecular and subcellular levels were performed with in-vitro reconstitution assays or in cell culture systems (Blanchoin et al., 2000; Pernier et al., 2016; Shekhar and Carlier, 2017); the importance of capping protein at the tissue level is not yet fully characterized.

Force generated by actomyosin machinery is essential for cell and tissue morphogenesis as well as the physiological function of many tissues (Agarwal and Zaidel-Bar, 2019). One example is the syncytial germline of $C$. elegans, where inward contraction of a corset-like actomyosin structure lining the rachis balances the outward pulling force of germ cell membrane tension (Priti et. al, 2018). Whether CP contributes to actin architecture and indirectly affects actomyosin contractility has not yet been investigated in an in-vivo system. Here, we explored the role of CP in the nematode C. elegans and discovered that it is critically important for maintaining a balanced level of actomyosin contractility in the germline, and is therefore required for the proper structure and function of the tissue.

\section{Results}

\section{CAP-1 is a component of the actomyosin corset in the syncytial germline}

To explore the expression pattern and sub-cellular localization of CAP-1 in the adult C. elegans hermaphrodite, we generated a strain in which CAP-1 is endogenously tagged with the fluorescent protein mKate 2 at its $\mathrm{N}$-terminus. Imaging by spinning disk confocal microscopy revealed that CAP-1 is expressed in multiple adult tissues, including the germline, spermatheca, pharynx, and vulva (Figure 1A). Co-staining of mKate2::CAP-1 worms with the $\mathrm{F}$-actin marker phalloidin showed that CAP-1 localizes exclusively to structures containing F- 
actin, and most, but not all, F-actin structures contain CAP-1 (Supplementary figure 1A). CAP1 was observed to localize at the apical side of gut epithelial cells, facing the intestinal lumen, and it was found in striations along body-wall muscle cells and in the germline (Supplementary figure 1B). The C. elegans gonad, with two symmetrical U-shaped arms, contains a syncytium of germ cells, which first form sperm that is stored in the spermatheca and later form oocytes (Kimble and Crittenden, 2007). The germ cells exist as a syncytium, connected to a common germplasm-filled core called rachis via cytoskeletal rings known as rachis bridges (Bauer et al., 2021). In the germline, CAP-1 was expressed in all germ cells, where it localized to the cell cortices and surrounded the nuclei, and in the germline syncytium CAP-1 was enriched at rachis bridges (Figure 1B). CAP-1 was also observed in the cortices of early embryos, the significance of which will be explored elsewhere. Here, we have focused on the function of CAP-1 in the germline.

We have previously shown that the syncytial germline architecture is maintained by contractility of an actomyosin corset surrounding the rachis (Priti et. al. 2018). To further investigate the localization of CAP-1 with respect to other components of the actomyosin corset, we crossed the mKate2::CAP-1 strain with strains expressing endogenously GFPtagged PLST-1/plastin, NMY-2/non-muscle myosin II, CYK-1/diaphanous formin, and ARX2/Arp2. As evident from the images and corresponding intensity line profiles, CAP-1 nearly perfectly co-localized with the actin cross-linker PLST-1 and NMY-2 along the rachis (Figure 1C, C', C'). CAP-1 and the formin CYK-1 also exhibited a high degree of correlation along the rachis, although their relative intensities fluctuated (Figure 1C, C', C''). The Arp2/3 complex, represented by ARX-2, appeared in punctate structures along the rachis bridges, and CAP-1 shows strong co-localization with ARX-2 at the punctate structures (Figure 1C, C', C'"). Thus, CAP-1 is an integral component of the actomyosin corset in the syncytial germline, associating with actomyosin as well as nucleators of both branched and linear F-actin.

\section{CAP-1 is required for maintenance of the germline architecture}

Given the prominent localization of CAP-1 in the actomyosin corset, we sought to understand its role in the germline through loss of function experiments. We generated a cap-1 null mutant by using CRISPR-Cas-9 to delete the entire cap- 1 gene. The homozygous null mutants, obtained from heterozygous mothers, successfully accomplish embryogenesis but arrest at the L3 larval stage. Since the homozygous cap-1 mutants did not reach adulthood they were not useful for our study of CAP-1 in the germline and we therefore used RNAi-mediated knockdown (KD).

To assess the degree of KD in the germline, we carried out cap-1 KD on worms expressing mKate $2::$ CAP-1 and a membrane marker (GFP::PLC1 $\delta$-PH) (Supplementary figure 2A). We observed a $\sim 60 \%$ decrease in CAP-1 fluorescence in the cap-1(RNAi) germlines as compared to control worms (Supplementary figure $2 \mathrm{~B}$ ). Next, we subjected worms co-expressing the membrane marker and nuclear marker (HIS-58::mCherry) to cap-1(RNAi) starting from larval stage 1 (L1) and assessed their germline architecture in adulthood. We observed a range of phenotypes, which we classified broadly as 'mild' (53\%) or 'severe' (47\%). As shown in Figure 2A, CAP-1 loss of function led to multiple defects in gonad structure, including an 
abnormal loop region, abnormally sized oocytes (some multinucleated), defects in rachis tube morphology, and loss or collapse of germ-cell membranes. While in control germlines all the nuclei are held within the germ cells, we found nuclei floating in the rachis tube in the majority of cap-1(RNAi) gonads, both mild and severe (Figure 2B). Furthermore, while each germ cell has only one nucleus in the control, we found multinucleated cells in $9 \% \pm 3 \%$ of mild and $27 \% \pm 1.8 \%$ of severe cap $-1(R N A i)$ germlines (Figure $2 \mathrm{C}$ ).

To determine the effect of CAP-1 loss of function on rachis morphology with higher precision, we repeated the KD in a strain expressing a membrane marker (mCherry::PLC1 $\delta$-PH) and the anillin isoform ANI-2 tagged with GFP to mark the rachis bridges (Amini et. al. 2015). Unlike the rachis of control gonads, which was straight and maintained a fairly constant diameter, the rachis of cap-1 (RNAi) worms was meandering and of variable diameter (Figure 2D, E). We quantified rachis straightness by dividing the length of a straight line between the two ends of the rachis by the length of a path along the centre of the rachis. In control worms, the rachis straightness was close to one, but it was significantly reduced upon cap-1 KD (Figure 2F). Next, we measured the ratio between rachis and gonad diameters. In gonads mildly affected by cap-1(RNAi) the mean diameter ratio was not different from control, but the variance was higher. The severely affected gonads, on the other hand, exhibited a severely constricted rachis as compared with the control (Figure $2 \mathrm{G}$ ). The decrease in rachis width is accompanied by an increase in germ cell height in the case of gonads severely affected by cap-1RNAi (Figure $2 \mathrm{H})$. For an overview of the various phenotypes appearing in cap-1(RNAi) germlines we analysed 48 worms expressing a membrane marker and found that over $80 \%$ had abnormal oocytes and an abnormal loop region, over $70 \%$ had rachis defects, and $\sim 30 \%$ exhibited germ cell membrane loss (Figure 2I).

To determine whether the germline defects observed are due to cap- 1 depletion in the germline or whether cap-1 depletion in other tissues might contribute to the phenotype, we performed cap-1(RNAi) in a germline-specific RNAi strain (DCL569), in which a null mutation in the RNAi-essential gene $r d e-1$ is rescued only in the germline by expression of $r d e-1$ under a germline-specific promoter (Zou et al., 2019). Following cap-1(RNAi) in this strain we observed the same germline defects as we found previously with whole worm RNAi and these defects appeared with the same penetrance and range of severities, suggesting that cap-1 depletion within the germline is sufficient to generate these structural defects (Supplementary figure $2 \mathrm{C}$ ).

To establish whether CAP-1 function is required during germline morphogenesis or for maintenance of its structure, we followed germline formation in cap-1(RNAi) conditions from the L2 stage until adulthood (Figure 2J). We did not observe any defect in gonad morphology during any of the larval stages. Starting from the young adult stage we could observe the onset of mild defects in gonad morphology in cap-1 depleted worms, mostly oocyte morphology defects, and the defects increased in severity with time resulting in further gross structural defects in the adult germline. These results suggest that CAP-1 is not required during gonad development but is essential for maintenance of its structure in adulthood.

\section{CAP-1 depletion leads to increased F-actin and ARX-2 in the germline}


To understand the dependence of germline structure on CAP-1 function we first set out to determine the effect of cap-1(RNAi) on the actin cytoskeleton. For this purpose, we chose to examine worms with a mild phenotype, because germline structure in the severely affected worms was too disrupted to compare with controls. F-actin, as visualized by phalloidin staining, is specifically enriched at rachis bridges in the germline, but is also observed at the basal and lateral sides of the germ cells, presumably marking their cortices. Phalloidin staining of dissected gonads showed a significant increase in F-actin in the germline of cap-1(RNAi) worms compared to control worms (Figure 3A). Quantification of fluorescence intensities showed that F-actin increased by approximately 2-fold at the rachis, lateral and basal side of the germ cells (Figure 3B-D). A similar increase was also observed with the endogenously GFP-tagged F-actin bundling protein PLST-1 in live worms (Supplementary figure 3A-D). Fluorescence recovery after photobleaching (FRAP) experiments did not reveal a significant difference in the turnover of PLST-1::GFP at the rachis bridges of cap-1(RNAi) worms, as compared to control worms (Supplementary figure 3E and 3G-I).

Given the substantial increase in F-actin levels in the germline, we explored whether cap-1 KD affected the localization or levels of either of the known germline actin nucleators: the formin CYK-1 and the Arp2/3 complex. As shown earlier, CYK-1::GFP is enriched at the rachis, where it co-localized with CAP-1 (Figure 1E). We did not observe any difference in the localization or levels of CYK-1 in the germline following cap-1(RNAi) (Figure 3E, G). In contrast, we observed an average 1.3-fold increase in the levels of the Arp2/3 component ARX2 in cap-1(RNAi) germlines (Figure 3F). The increase in ARX-2 was observed throughout the germline as well as within the punctate structures found along the rachis (Figure $3 \mathrm{H}, \mathrm{I}$ ).

\section{CAP-1 depletion leads to an increase in active NMY-2 at the germline corset}

Given the increase in F-actin in the rachis following cap- 1 depletion and the known dependence of syncytial germline architecture on rachis actomyosin contractility, we investigated the effect of cap-1(RNAi) on non-muscle Myosin II/NMY-2 at the rachis. As shown in Figure 4A, we observed a 2-fold increase in NMY-2 levels at the rachis in cap-1(RNAi) (Figure 4A, B). Furthermore, using an antibody that recognizes only the active/phosphorylated form of Myosin II, we also observed an increase in the levels of phospho-myosin II at the rachis (Figure 4C, D), indicating an increase in contractility of the actomyosin corset in cap-1(RNAi) conditions. The observed increase in NMY-2 could, in theory, be due to more F-actin binding sites, to slower NMY-2 turnover, or a combination of both. In order to examine if the dynamics of NMY-2 were affected by cap-1 depletion we carried out Fluorescence recovery after photo bleaching (FRAP) on endogenously tagged NMY-2::GFP at the rachis bridge. We did not observe a significant difference in the recovery kinetics of NMY-2 in cap-1 (RNAi) versus the control, although we did observe a trend towards a larger immobile fraction in cap-1 depleted background (Supplementary figure 3F, J, K, L). Thus, the increase in NMY-2 appears to be mainly due to the increase in F-actin.

\section{Increased actomyosin contractility can explain the germline defects of CAP-1 loss of function}

The observed increase in F-actin and active NMY-2 at the rachis led us to hypothesize that cap-1 loss of function phenotypes is due to increased contractility of the rachis actomyosin 
corset. To test this idea, we first compared germline morphology of mel-11(RNAi) with cap1(RNAi). MEL-11 is the $C$. elegans myosin phosphatase, and its depletion is known to lead to an increase in actomyosin contractility (Piekny and Mains, 2002). Importantly, we observed similar morphological changes, namely a constricted rachis and increased germ cell height in cap-1(RNAi) and mel-11(RNAi) (Supplementary figure 3M). If indeed cap-1 germline defects are due to increased rachis contractility, then reducing myosin activity should rescue these defects. We tested this idea by depleting the myosin activator Rho kinase/LET-502 in cap1(RNAi) worms. As evident in figure 4E, let-502(RNAi) displayed a phenotype opposite of that of mel-11(RNAi) or cap-1(RNAi), namely it had a wider than normal rachis and shorter germ cell membranes. We performed the double RNAi KD in worms expressing mKate2::CAP-1 and quantified CAP-1 intensity to confirm cap- 1 depletion and rule out a dilution effect (Figure 4E and 4F). Germlines with the double KD of let-502 and cap-1 resembled the let-502 (RNAi) phenotype of a broad rachis and short germ cell membranes, indicating that the morphological defects observed in cap-1(RNAi) gonads are a result of increased contractility of the actomyosin corset in the syncytial germline (Figure $4 \mathrm{G}, \mathrm{H}$ ).

\section{Discussion}

Tissue morphogenesis involves coordination between multiple cells undergoing structural and identity changes and generating pushing and pulling forces against each other to ultimately assume a functional shape (Heer and Martin, 2017). Tissue homeostasis is also dependent on a fine balance between external and internal mechanical forces. Here, we found actin CP CAP1 to be a critical component of the $C$. elegans germline rachis actomyosin corset, where it colocalizes with F-actin as well as formin and Arp2/3 and regulates its contractility.

Different actin structures varying in architecture, connectivity and contractility can exist within the same cell or tissue and accomplish different functions in a spatiotemporally regulated manner. For example, the fission yeast cytoskeleton has four distinct actin structures, which differ largely from each other in structure, function and mechanical properties (Kovar et al., 2011). CP associates with actin patches in the fission yeast to exclude formin from the Arp $2 / 3$ complex-mediated actin structures, hence preserving the identity of distinct actin structures (Billault-Chaumartin and Martin, 2019). We found CAP-1 in the syncytial C. elegans germline to be enriched at rachis bridges, which differ from the rest of the germ cell cortices in terms of composition and function (Amini et al., 2014; Priti et al., 2018). The formin, CYK-1 exhibits an enriched localisation at the rachis bridge while the Arp2/3 component ARX-2 does not, suggesting a differential distribution of branched and linear actin networks in the germline. However, we did not observe spreading of formin into Arp2/3 domains in the absence of CAP1. Instead, we observed an increase in F-actin and, to a lesser degree, an increase in ARX-2 levels everywhere in the germline. The increase in F-actin can be explained by the absence of a factor stopping its polymerization. The mechanism responsible for increasing ARX-2 is not clear and may be due to an as-of-now uncharacterized transcriptional response or simply by the availability of more F-actin. Nevertheless, since the increase in F-actin exceeded the increase in ARX-2 levels, the density of branched actin in the network likely decreased, altering the balance between linear and branched actin in favour of linear actin. We therefore propose that loss of CAP-1 results in a change in the architecture of the actin network at the rachis 
bridge, which supports a common role for CAP-1 in generating and maintaining distinctly different actin structures in a cell or tissue.

Actin and non-muscle myosin II combine to form contractile structures, which are regulated spatiotemporally by several accessory proteins. Multiple actin binding proteins, including filamin, fascin, anillin, ezrin, and moesin, have been shown to affect actomyosin contractility via regulation of different aspects of actin organisation (Murrell et al., 2015). We found a dramatic increase in NMY-2 levels at the rachis upon CAP-1 KD, a result that was confirmed by phospho-myosin II staining. Our FRAP experiments did not detect a significant change in NMY-2 dynamics following CAP-1 KD. On the other hand, the twofold increase in NMY-2 levels at the rachis closely matches the twofold increase in F-actin following CAP-1 KD. We therefore conclude that the increase in myosin levels is primarily due to the availability of more F-actin.

The balance between linear and branched actin can also influence the contractility of the system as a whole. In-vitro reconstitution assays and modelling have shown that the balance between linear and branched actin networks influences the level of 'network connectivity', which can affect myosin mediated contractility according to a bell-shaped curve (Belmonte et al., 2017; Ennomani et al., 2016). We do not know where along this connectivity curve the rachis actomyosin network is in wild-type conditions, but it is possible that a decrease in connectivity due to the decreased density of Arp $2 / 3$ branching results in increased contractility. Alternatively, the increase in contractility upon cap-1 depletion might be despite the decrease in connectivity and is solely due to the increase in linear F-actin and NMY-2.

The consequences for the germline of increased rachis contractility due to CAP-1 loss of function are disastrous for both the structure and function of the tissue. While development of the germline proceeds normally in the absence of CAP-1, maintenance of the syncytial germline structure requires CAP-1 and in its absence multiple structural defects appear. The increased contractility results in a highly constricted rachis, accompanied by defective oocytes. Importantly, inhibiting actomyosin contractility in the CAP-1 KD condition, by inhibiting myosin phosphorylation by Rho Kinase, is able to rescue the restricted rachis phenotype, strengthening our conclusion that CAP-1 affects rachis morphology through actomyosin contractility. Actin CP has been shown to affect actin organisation in Drosophila bristles and oocytes (Gates et al., 2009; Hopmann and Miller, 2003). Studies in cells have highlighted the importance of CP in cytoskeletal organisation and actin-driven protrusive activity (Fujiwara et al., 2014; Johnston et al., 2018; Jung et al., 2016). To the best of our knowledge, our study is the first to demonstrate a role for $\mathrm{CP}$ in regulating actomyosin contractility in a tissue. We found CAP-1 to be strongly expressed in multiple tissues in the adult worm and in the embryo. Future studies will reveal the role CP plays in other tissues and establish which are tissuespecific and which are general functions of $\mathrm{CP}$ in vivo.

\section{Acknowledgements}

This study was supported by Israel Science Foundation grant 767/20 awarded to RZB, and scholarships from the Mechanobiology Institute Singapore and the Israeli Council for higher 
education awarded to S.R. Some strains were provided by the CGC, which is funded by NIH Office of Research Infrastructure Programs (P40 OD010440). We thank Edwin Munro and David Kovar (The University of Chicago) for illuminating discussions.

\section{Author contributions}

Conceptualization, S.R., P.A. and R.Z.B; Investigation, S.R. and P.A.; Formal Analysis, S.R. and P. A.; Visualization, S.R.; Writing - Original Draft, S.R; Writing - Review \& Editing, P.A. and R.Z.B.; Supervision, R.Z.B.; Funding Acquisition, R.Z.B.

\section{Declaration of interests}

The authors declare no competing interests.

\section{Figure legends}

Figure 1: CAP-1 localizes at actomyosin-enriched structures in the $C$. elegans germline. (A) Inverted grayscale confocal fluorescence image of an adult C. elegans hermaphrodite expressing CAP-1endogenously tagged with mKate2. Arrows point to spermatheca and embryos. Arrowheads indicate germline. Scale bar, $20 \mu \mathrm{m}$.

(B) Representative inverted grayscale confocal fluorescence images of the C. elegans germline expressing mKate2::CAP-1. Midplane view showing enrichment of CAP-1 at rachis bridges and nuclei of germ cells, basal plane view showing localization at the germ cell cortex (arrowheads) and a maximum intensity Z-projection view showing CAP-1-enriched actomyosin corset in the gonad. Scale bar, $20 \mu \mathrm{m}$. Inset scale bar, $5 \mu \mathrm{m}$.

(C) Midplane view of the C. elegans germline showing colocalization of mKate2::CAP-1 with PLST-1::GFP, NMY-2::GFP, CYK-1::GFP and ARX-2::GFP. Scale bar, $20 \mu \mathrm{m}$.

(C') Magnified view of the rachis region marked by a white rectangle in panel C. Scale bar, $5 \mu \mathrm{m}$.

(C') Fluorescence intensity profiles of endogenously-tagged proteins along a 5-pixel wide line drawn along the rachis between the arrowheads marked in panel C'.

Figure 2: CAP-1 is required for the maintenance of germline architecture and functionality.

(A) Confocal fluorescence images of the C. elegans germline expressing a membrane marker, GFP::PLC1 $\delta$-PH and a nuclear marker, HIS-58::mCherry. Top row shows control germlines while middle and bottom rows show cap-1(RNAi) germlines with mild and severe phenotypes. Arrowheads show nuclei mispositioned within the rachis. Arrows indicate multinucleated germ cells.

$(B, C)$ Percentage of gonads showing the phenotypes 'nuclei in rachis' and 'multinucleated germ cells' in gonads of control $(\mathrm{N}=32)$ versus mild $(\mathrm{N}=15)$ and severely affected $(\mathrm{N}=15)$ cap1(RNAi) worms.

(D) Confocal fluorescence images of the midplane and Z-projected views of gonads expressing a membrane marker, mCherry::PLC1 $\delta$-PH and a rachis bridge marker, ANI-2::GFP for control (top), mildly (middle) and severely (bottom) affected cap-1(RNAi) worms. Arrowheads 
indicate abnormal loop region (blue), abnormal oocytes (yellow), constricted and meandering rachis (pink) and membrane loss (orange).

(E) Magnified views of the regions marked by white boxes in panel D, showing rachis morphology in gonads of control and cap-1(RNAi) worms.

(F, G, H) Quantification of rachis straightness, rachis width and germ cell height in the gonads of control $(\mathrm{N}=38)$ versus mild $(\mathrm{N}=19)$ and strong cap-1 (RNAi) $(\mathrm{N}=22)$ worms.

(I) Percentage of phenotypes observed in CAP-1 KD gonads $(\mathrm{N}=48)$.

(J) Confocal fluorescence images of germlines expressing the membrane marker (red) and ANI-2::GFP (cyan) from larval stage 2 till adulthood in control and cap-1(RNAi) worms.

Asterisks indicate the proximal end of the germlines. Arrows and arrowheads indicate defective oocytes and rachis, respectively in cap-1(RNAi). Scale bars, $20 \mu \mathrm{m}$.

Error bars are \pm SEM. ${ }^{* *}$ p value $<0.01 ; * * *$ p value $<0.001 ;$ ns, not significant (Student's ttest).

Figure 3: CAP-1 regulates F-actin and ARX-2::GFP levels in the $C$. elegans germline.

(A) Midplane, basal plane and maximum-intensity Z-projected images of phalloidin stained germlines in control and cap-1(RNAi) worms. Scale bar, $20 \mu \mathrm{m}$.

(B, C, D) Quantification of mean F-actin intensity in untreated control $(\mathrm{N}=52)$ versus cap1(RNAi) $(\mathrm{N}=42)$ at rachis bridges (arrows in $\mathrm{A})$, lateral membranes (arrowheads in $\mathrm{A}$ ) and basal sides of the germ cells (box in A).

(E, F) Confocal fluorescence images of germlines expressing CYK-1::GFP and ARX-2::GFP in control versus cap-1(RNAi) worms. Scale bar, $20 \mu \mathrm{m}$.

(E', F') Magnified view of the rachis region marked by a yellow rectangle in panels $\mathrm{E}, \mathrm{F}$, respectively. Scale bar, $5 \mu \mathrm{m}$.

(G) Quantification of CYK-1::GFP intensity at the rachis bridges in control $(\mathrm{N}=41)$ versus cap1(RNAi) $(\mathrm{N}=40)$ worms.

$(\mathrm{H}, \mathrm{I})$ Quantification of ARX-2::GFP intensity in control versus cap-1(RNAi) worms within the whole germline $(\mathrm{N}=40,25)$ and at the rachis $(\mathrm{N}=14,14)$, respectively.

Error bars are \pm SEM. ${ }^{* * *}$ p value $<0.001 ;$ ns, not significant (Student's t-test).

\section{Figure 4: CAP-1 regulates contractility to maintain germline structure}

(A) Confocal images of the germline expressing NMY-2::GFP (cyan) and GFP::PLC1 $\delta$-PH (red) in control and cap-1(RNAi) worms. Scale bar, $20 \mu \mathrm{m}$.

(B) Quantification of NMY-2::GFP intensity at the rachis bridge in control $(\mathrm{N}=23)$ versus cap1(RNAi) (N=24) worms. Error bars represent \pm SEM. ****p value $<0.0001$ (Student's t-test). Scale bar, $20 \mu \mathrm{m}$.

(C) Confocal images of the germline in control and cap-1(RNAi) worms stained for phosphorylated Myosin II (pMyoII, cyan) and F-actin (phalloidin, red). Scale bar, $20 \mu \mathrm{m}$.

(D) Quantification of phospho-myosin II intensity at the rachis bridge in control $(\mathrm{N}=35)$ versus cap-1 (RNAi) $(\mathrm{N}=31)$ worms. Error bars represent $\pm \mathrm{SEM}$. ****p value $<0.0001$ (Student's $\mathrm{t}-$ test). Scale bar, $20 \mu \mathrm{m}$

(E) Midplane confocal views of the whole germline and magnified views of the rachis region (white box) in worms expressing PLST-1::GFP (cyan) and mKate2::CAP-1 (red) in control, 
cap-1(RNAi), let-502(RNAi) and cap-1(RNAi);let-502(RNAi). Double-headed arrows mark rachis diameter. Scale bar, $20 \mu \mathrm{m}$, scale bar in magnified images, $5 \mu \mathrm{m}$.

$(\mathrm{F}, \mathrm{G}, \mathrm{H})$ Quantification of mKate2::CAP-1 intensity, rachis width and germ cell height in germlines of control (N=28), cap-1(RNAi) ( $\mathrm{N}=23)$, let-502(RNAi) $(\mathrm{N}=31)$ and cap1(RNAi);let-502(RNAi) ( $\mathrm{N}=29)$ worms.

Error bars are \pm SEM. ${ }^{*}$ p value $<0.1 ; * *$ p value $<0.01$; ****p value $<0.0001$ (One-way ANOVA test).

\section{Materials and Methods}

\section{Worm strain maintenance}

All C. elegans strains were grown and maintained on NGM (nematode growth medium) plates seeded with OP50 bacteria according to standard protocols (Brenner, 1974). All worms were kept at $20^{\circ} \mathrm{C}$, unless specified otherwise. All strains used in this study have been listed in the table. Some strains in this study have been obtained from the CGC (University of Minnesota) and some have been made by InVivo Biosystems (Eugene, OR, USA) using CRISPR-Cas9 knock-in technology.

\begin{tabular}{|c|c|c|}
\hline Strain & Genotype & Source \\
\hline COP1725 & $\begin{array}{l}\text { cap-1 (knu650 [pNU1550 - N-terminal degron mKate2 unc- } \\
119(+)]) ; \text { unc-119(ed3) III }\end{array}$ & $\begin{array}{l}\text { InVivo } \\
\text { Biosystems }\end{array}$ \\
\hline RZB406 & $\begin{array}{l}\text { cap-1 (knu650 [pNU1550 - N-terminal degron mKate2 unc- } \\
\text { 119(+)]) ; unc-119(ed3) III; ItIs38[pAA1; pie- } \\
1:: G F P:: P H(P L C 1 d e l t a 1)+\text { unc-119(+)] }\end{array}$ & This study \\
\hline RZB355 & $\begin{array}{l}\text { cap-1 (knu650 [pNU1550 - N-terminal degron mKate2 unc- } \\
119(+)]) ; \text { unc-119(ed3) III; plst-1(msn190[plst-1::gfp]) IV }\end{array}$ & This study \\
\hline RZB347 & $\begin{array}{l}\text { cap-1 (knu650 [pNU1550 - N-terminal degron mKate2 unc- } \\
119(+)]) ; \text { cyk-1 (knu83 C-terminal GFP, unc-119 (+)); unc- } \\
119(\text { ed3) III }\end{array}$ & This study \\
\hline RZB348 & $\begin{array}{l}\text { cap-1 (knu650 [pNU1550 - N-terminal degron mKate2 unc- } \\
119(+)]) ; \text { cas607[arx-2::gfp knock-in] V) }\end{array}$ & This \\
\hline RZB400 & $\begin{array}{l}\text { cap-1 (knu650 [pNU1550 - N-terminal degron mKate2 unc- } \\
119(+)] ; \text { nmy-2(cp13[nmy-2::gfp }+ \text { LoxP]) I. })\end{array}$ & This study \\
\hline RZB266 & $\begin{array}{llccc}\text { nmy-2 (cp52[nmy-2::mkate] }) \quad I ; t I s 38 & \text { [pAA1; } & \text { pie- } \\
1: \because G F P:: P H(P L C 1 \text { delta1 })+u n c-119(+)] . & & \\
\end{array}$ & (Priti et al., 2018) \\
\hline RZB220 & plst-1(msn190[plst-1::gfp]) IV; pie-1:mChe::PH & This study \\
\hline LP162 & $n m y-2(c p 13[n m y-2: \because g f p+L o x P]) I$. & CGC \\
\hline UM208 & $\begin{array}{l}\text { unc-119(ed3) III; ltIs81 [Ppie-1::gfp-TEV-Stag::ani-2; unc- } \\
119(+)] ; \text { ltIs44 [Ppie-1::mCherry::PH(PLC1delta1); unc- } \\
119(+)] I V\end{array}$ & $\begin{array}{l}\text { (Amini et al., } \\
\text { 2014) }\end{array}$ \\
\hline OD95 & $\begin{array}{l}\text { Itls37 [pAA64; pie-1::mCherry }:: H I S-58+u n c-119(+) . \text { Itls38 } \\
{[p A A 1 ; \text { pie-1::GFP::PH(PLC1delta1)+unc-119(+)] }}\end{array}$ & $\begin{array}{l}\text { (Amini et al., } \\
\text { 2014) }\end{array}$ \\
\hline DCL569 & mkcSi13 [sun-1p::rde-1::sun-1 3'UTR + unc-119(+)] II & (Zou et. al., 2019) \\
\hline
\end{tabular}




\section{RNA interference}

All RNAi experiments were performed by feeding RNAi. Bacterial clones of HT115(DE3) bacterial strain expressing the vector L4440 containing gene specific sequences were obtained from the Ahringer or Vidal libraries (Source BioScience) and sequenced for confirmation. RNAi plates were prepared with NGM media containing $1 \mathrm{mM}$ IPTG and $100 \mu \mathrm{g} / \mathrm{ml}$ of ampicillin. A bacterial clone containing L4440 vector alone was used as a negative control for the experiments, unless specified otherwise. For dsRNA induction, an overnight culture of bacteria was diluted 1:50 and grown at $37^{\circ} \mathrm{C}$ with shaking for 3-4 hours, after which IPTG was added to a final concentration of $1 \mathrm{mM}$ and allowed to grow for an additional 3-4 hrs. This culture was used to seed the RNAi plates, air dried and grown overnight at room temperature. Gravid hermaphrodites were bleached on the RNAi plates to release the embryos (unless specified otherwise). Worms hatched on the RNAi plate were grown to adulthood, when they were collected either for immunofluorescence or live imaging. UM208 mild phenotype exhibiting worms were put on the RNAi plates at L3 stage and grown till adulthood.

\section{Phalloidin staining}

Gonads were dissected in M9 buffer containing $0.5 \mathrm{mM}$ levamisole on a glass slide. The dissected gonads were fixed in 3.5\% formaldehyde in PBS for 20 minutes, then permeabilised in PBS with $0.025 \%$ Triton-X100 for 5 minutes. Next, the gonads were incubated in phalloidinTRITC (Sigma, P1951) in the dark at room temperature. The gonads were washed with PBS to remove excess phalloidin and were mounted on a glass slide with vectashield mounting medium (Vector lab, Cat. No. H-1000) and mounted for imaging.

\section{Immunofluorescence}

Dissected gonads (in $0.5 \mathrm{mM}$ levamisole in M9 buffer) were washed to remove levamisole and fixed with 3.5\% formaldehyde in PBS for 20 minutes. Next, the gonads were washed thrice in PBS and then permeabilised with $0.25 \%$ tween in PBS for 10 mins. The gonads were then washed thrice with PBS, incubated in blocking solution (1\% BSA, 0.1\% Tween and 30mM glycine in PBS) at room temperature for 1 hour. A 1:400 dilution of anti phosphoMLC Ser19 (Cell Signaling Technology, Cat. no. 3671) in blocking solution was used to incubate the gonads at $4^{\circ} \mathrm{C}$ overnight. After 3 washes with PBS, the gonads were incubated with a solution containing 1:500 dilution of anti-rabbit secondary antibody conjugated with Alexa 488 (Invitrogen, Cat. no. A21244), 1:250 phalloidin-TRITC (Sigma, Cat. no. P1951) and 1:1000 DAPI (Sigma-Aldrich) in blocking buffer at room temperature for 1.5 hours. After 3 washes with PBS, the gonads were stored in vectashield (Vector lab, Cat. no. H-1000) and mounted for imaging.

\section{Microscopy and image acquisition}

Live adult hermaphrodites in $10 \mathrm{mM}$ levamisole or immunostained dissected gonads stored in vectashield were mounted on fresh $3 \%$ agarose pads prepared on a glass slide. Imaging was carried out with a Nikon Ti2E microscope equipped with a Yokogawa W1 spinning disk system and a Plan Apo 60X oil 1.4 NA and a Plan Apo 100X oil 1.45 NA. Samples were illuminated with $405 \mathrm{~nm}$, $488 \mathrm{~nm}$ or $561 \mathrm{~nm}$ lasers (Gataca systems, France) for excitation and acquired on a prime 95B sCMOS camera (Photometrics, Tucson, AZ). The software Metamorph version 7.10.2.240 (Molecular Devices, CA) was used as the controlling interface. All images were captured with $\mathrm{Z}$ stacks of $1 \mu \mathrm{m}$ spacing.

\section{Image analysis}


All image analysis was carried out with the help of FIJI (Schindelin et al., 2012). For measurement of germ cell height, several germ cells were selected in the pachytene region in the $\mathrm{Z}$ plane exhibiting the germline midplane. Germ cell height was measured for selected cells. The ratio of germline width/rachis width was measured at three regions in the germline midplane and the average ratio was calculated for each gonad.

Line scan intensity plot: to analyse the relative localisation between mKate2::CAP-1 and PLST-1::GFP, NMY-2::GFP, ARX-2::GFP or CYK-1::GFP at the rachis, a 5 pixel thick line was drawn along the rachis and the fluorescence profile was generated for both channels with Fiji and graphs were plotted with GraphPad Prism 9.

Fluorescence intensity measurement: each image was corrected for the background fluorescence before measurement. The mid plane of the germline rachis was selected for analysis of PLST-1::GFP, CYK-1::GFP, NMY-2::mKate and phalloidin at the rachis bridges using Fiji. Mean intensity was measured along a 5-pixel wide line drawn along the rachis bridges for both control and RNAi conditions. For measurement of PLST-1::GFP and phalloidin at the basal and lateral plane, the basal and lateral plane of the germline was selected respectively. The segmented line tool was used to trace the cell boundaries at the basal plane and the cell junctions at the lateral plane and mean intensity was calculated.

Total ARX-2::GFP fluorescence was calculated by tracing the germline outline at the midplane and mean intensity was measured after background subtraction. For measurement of ARX-2 puncta fluorescence, the rachis midplane was outlined and thresholded for selecting the puncta and the mean intensity was measured.

Fluorescence recovery after photobleaching (FRAP): FRAP experiments were performed with the iLAS2 module (Gataca systems, Massy, France) for targeted laser illumination on the spinning disk microscope described above with a Plan Apo 100X oil 1.4 NA objective. A circular region of interest along the germline rachis of worms expressing NMY-2::GFP was selected manually and photobleached with the $488 \mathrm{~nm}$ laser at $80-100 \%$ laser power. Images with five $\mathrm{Z}$ slices ( $1 \mu \mathrm{m}$ spacing) were acquired using $50 \% 488 \mathrm{~nm}$ laser and exposure time of $400 \mathrm{~ms}$ at an interval of 30 secs for a total duration of 10 mins. The average intensity of the ROI was measured with Fiji for the bleached, unbleached and background ROIs. The fluorescence intensity of the bleached region was double normalised for photobleaching and background using the double normalisation method. The final analysis of difference in recovery rates was done with Graphpad Prism 9 as performed in (Priti et al., 2018).

\section{Statistical analysis}

All statistical analysis was carried out with the software Graphpad Prism 9. Student's t-test or one-way analysis of variance (ANOVA) was used to carry out tests of significance for two samples or more than two samples, respectively. All graphs are represented as mean \pm S.E. The sample number for each experiment is indicated in the corresponding figure legend. 


\section{References}

Agarwal, P., and Zaidel-Bar, R. (2019). Principles of Actomyosin Regulation In Vivo. Trends Cell Biol. 29, 150-163.

Akin, O., and Mullins, R.D. (2008). Capping Protein Increases the Rate of Actin-Based Motility by Promoting Filament Nucleation by the Arp2/3 Complex. Cell 133, 841-851. Amini, R., Goupil, E., Labella, S., Zetka, M., Maddox, A.S., Labbé, J.-C., and Chartier, N.T. (2014). C. elegans Anillin proteins regulate intercellular bridge stability and germline syncytial organization. J. Cell Biol. 206, 129-143.

Bauer, J., Poupart, V., Goupil, E., Nguyen, K.C.Q., Hall, D.H., and Labbé, J.-C. (2021). The initial expansion of the $\mathrm{C}$. elegans syncytial germ line is coupled to incomplete primordial germ cell cytokinesis. Development 148 .

Bear, J.E., and Gertler, F.B. (2009). Ena/VASP: towards resolving a pointed controversy at the barbed end. J. Cell Sci. 122, 1947-1953.

Belmonte, J.M., Leptin, M., and Nédélec, F. (2017). A theory that predicts behaviors of disordered cytoskeletal networks. Mol. Syst. Biol. 13, 941.

Billault-Chaumartin, I., and Martin, S.G. (2019). Capping Protein Insulates Arp2/3Assembled Actin Patches from Formins. Curr. Biol. 29, 3165-3176.e6.

Blanchoin, L., Pollard, T.D., and Mullins, R.D. (2000). Interactions of ADF/cofilin, Arp2/3 complex, capping protein and profilin in remodeling of branched actin filament networks. Curr. Biol. 10, 1273-1282.

Bombardier, J.P., Eskin, J.A., Jaiswal, R., Corrêa, I.R., Xu, M.Q., Goode, B.L., and Gelles, J. (2015). Single-molecule visualization of a formin-capping protein "decision complex" at the actin filament barbed end. Nat. Commun. 6, 8707.

Brenner, S. (1974). The genetics of Caenorhabditis elegans. Genetics 77, 71-94.

Edwards, M., Zwolak, A., Schafer, D.A., Sept, D., Dominguez, R., and Cooper, J.A. (2014).

Capping protein regulators fine-tune actin assembly dynamics. Nat. Rev. Mol. Cell Biol. 15, 677-689.

Ennomani, H., Letort, G., Guérin, C., Martiel, J.-L., Cao, W., Nédélec, F., De La Cruz, E.M., Théry, M., and Blanchoin, L. (2016). Architecture and Connectivity Govern Actin Network Contractility. Curr. Biol. 26, 616-626.

Fujiwara, I., Remmert, K., Piszczek, G., and Hammer, J.A. (2014). Capping protein regulatory cycle driven by CARMIL and V-1 may promote actin network assembly at protruding edges. Proc. Natl. Acad. Sci. 111, E1970-E1979.

Funk, J., Merino, F., Schaks, M., Rottner, K., Raunser, S., and Bieling, P. (2021). A barbed end interference mechanism reveals how capping protein promotes nucleation in branched actin networks. Nat. Commun. 2021121 12, 1-17.

Gates, J., Nowotarski, S.H., Yin, H., Mahaffey, J.P., Bridges, T., Herrera, C., Homem, C.C.F., Janody, F., Montell, D.J., and Peifer, M. (2009). Enabled and Capping protein play important roles in shaping cell behavior during Drosophila oogenesis. Dev. Biol. 333, 90107.

Heer, N.C., and Martin, A.C. (2017). Tension, contraction and tissue morphogenesis. Hopmann, R., and Miller, K.G. (2003). A balance of capping protein and profilin functions is required to regulate actin polymerization in Drosophila bristle. Mol. Biol. Cell 14, 118-128. Iwasa, J.H., and Mullins, R.D. (2007). Spatial and temporal relationships between actinfilament nucleation, capping, and disassembly. Curr. Biol. 17, 395-406.

Johnston, A.B., Hilton, D.M., McConnell, P., Johnson, B., Harris, M.T., Simone, A., Amarasinghe, G.K., Cooper, J.A., and Goode, B.L. (2018). A novel mode of capping proteinregulation by twinfilin. Elife 7 .

Jung, G., Alexander, C.J., Wu, X.S., Piszczek, G., Chen, B.C., Betzig, E., and Hammer, J.A. (2016). V-1 regulates Capping Protein activity in vivo. Proc. Natl. Acad. Sci. U. S. A. 113, 
E6610-E6619.

Kim, T., Cooper, J.A., and Sept, D. (2010). The Interaction of Capping Protein with the Barbed End of the Actin Filament. J. Mol. Biol. 404, 794-802.

Kimble, J., and Crittenden, S.L. (2007). Controls of Germline Stem Cells, Entry into Meiosis, and the Sperm/Oocyte Decision in Caenorhabditis elegans. Annu. Rev. Cell Dev. Biol. 23, 405-433.

Kovar, D.R., Sirotkin, V., and Lord, M. (2011). Three's company: the fission yeast actin cytoskeleton. Trends Cell Biol. 21, 177-187.

Murrell, M., Oakes, P.W., Lenz, M., and Gardel, M.L. (2015). Forcing cells into shape: the mechanics of actomyosin contractility. Nat. Rev. Mol. Cell Biol. 16, 486-498.

Pernier, J., Shekhar, S., Jegou, A., Guichard, B., and Carlier, M.F. (2016). Profilin Interaction with Actin Filament Barbed End Controls Dynamic Instability, Capping, Branching, and Motility. Dev. Cell 36, 201-214.

Piekny, A.J., and Mains, P.E. (2002). Rho-binding kinase (LET-502) and myosin

phosphatase (MEL-11) regulate cytokinesis in the early Caenorhabditis elegans embryo. J.

Cell Sci. 115, 2271-2282.

Pollard, T.D. (2016). Actin and Actin-Binding Proteins. Cold Spring Harb. Perspect. Biol. 8, a018226.

Pollard, T.D., and Cooper, J.A. (2009). Actin, a central player in cell shape and movement.

Science (80-. ). 326, 1208-1212.

Priti, A., Ong, H.T., Toyama, Y., Padmanabhan, A., Dasgupta, S., Krajnc, M., and ZaidelBar, R. (2018). Syncytial germline architecture is actively maintained by contraction of an internal actomyosin corset. Nat. Commun. 9, 4694.

Schaub, S., Meister, J.-J., and Verkhovsky, A.B. (2007). Analysis of actin filament network organization in lamellipodia by comparing experimental and simulated images. J. Cell Sci. 120, 1491-1500.

Schindelin, J., Arganda-Carreras, I., Frise, E., Kaynig, V., Longair, M., Pietzsch, T., Preibisch, S., Rueden, C., Saalfeld, S., Schmid, B., et al. (2012). Fiji: an open-source platform for biological-image analysis. Nat. Methods 201297 9, 676-682.

Shekhar, S., and Carlier, M.F. (2017). Enhanced Depolymerization of Actin Filaments by ADF/Cofilin and Monomer Funneling by Capping Protein Cooperate to Accelerate BarbedEnd Growth. Curr. Biol. 27, 1990-1998.e5.

Shekhar, S., Kerleau, M., Kühn, S., Pernier, J., Romet-Lemonne, G., Jégou, A., and Carlier, M.-F. (2015). Formin and capping protein together embrace the actin filament in a ménage à trois. Nat. Commun. 6, 8730.

Shekhar, S., Pernier, J., and Carlier, M.-F. (2016). Regulators of actin filament barbed ends at a glance. J. Cell Sci. 129, 1085-1091.

Vinzenz, M., Nemethova, M., Schur, F., Mueller, J., Narita, A., Urban, E., Winkler, C., Schmeiser, C., Koestler, S.A., Rottner, K., et al. (2012). Actin branching in the initiation and maintenance of lamellipodia. J. Cell Sci. 125, 2775-2785.

Winder, S.J., and Ayscough, K.R. (2005). Actin-binding proteins. J. Cell Sci. 118, 651-654. Yamashita, A., Maeda, K., and Maéda, Y. (2003). Crystal structure of CapZ: structural basis for actin filament barbed end capping. EMBO J. 22, 1529-1538.

Zou, L., Wu, D., Zang, X., Wang, Z., Wu, Z., and Chen, D. (2019). Construction of a germline-specific RNAi tool in C. elegans. Sci. Rep. 9, 2354. 
bioRxiv preprint doi: https://doi.org/10.1101/2021.10.03.462551; this version posted October 3, 2021. The copyright holder for this preprint (which was not certified by peer review) is the author/funder. All rights reserved. No reuse allowed without permission.

Figure - 1
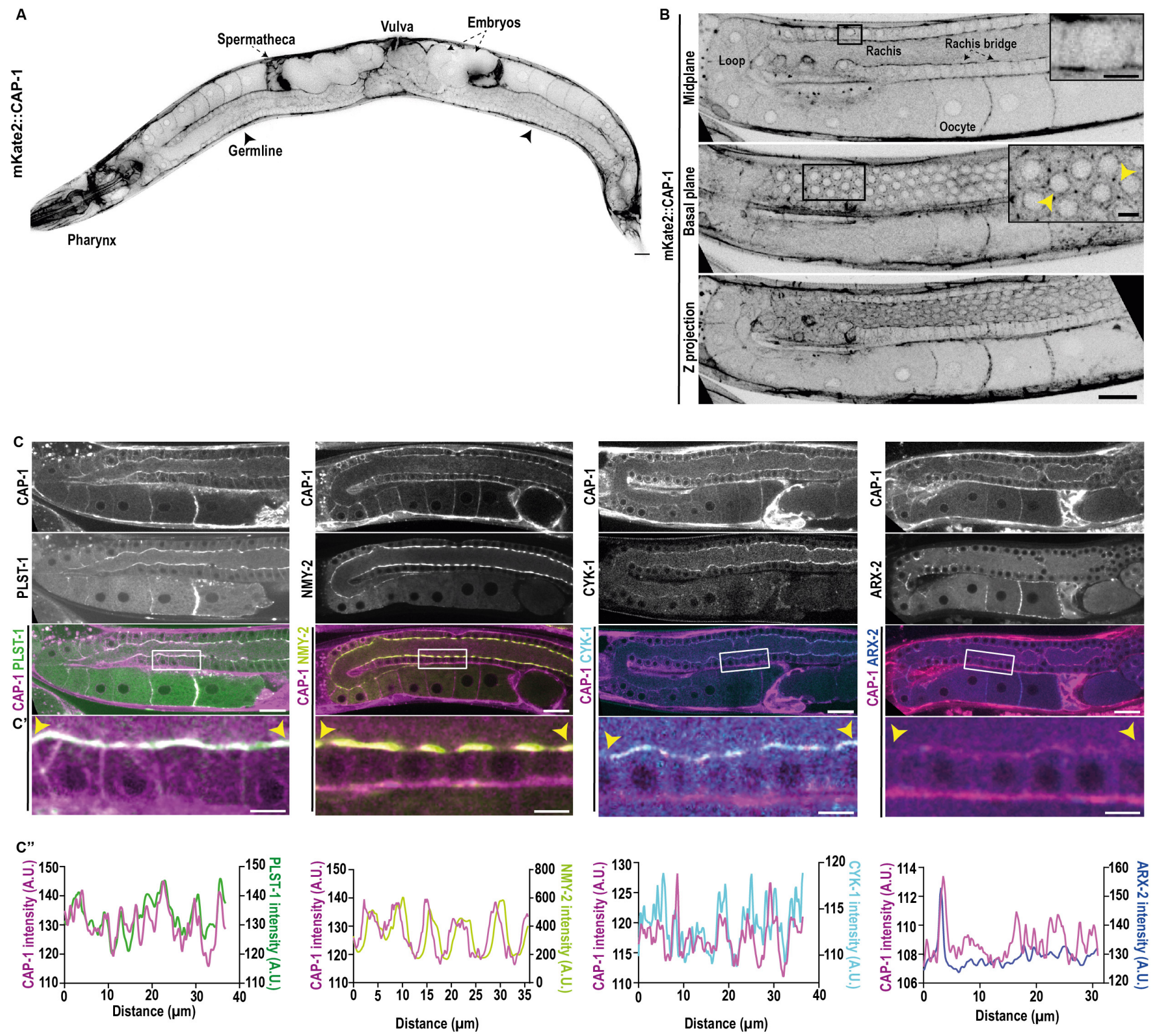
Figure -2
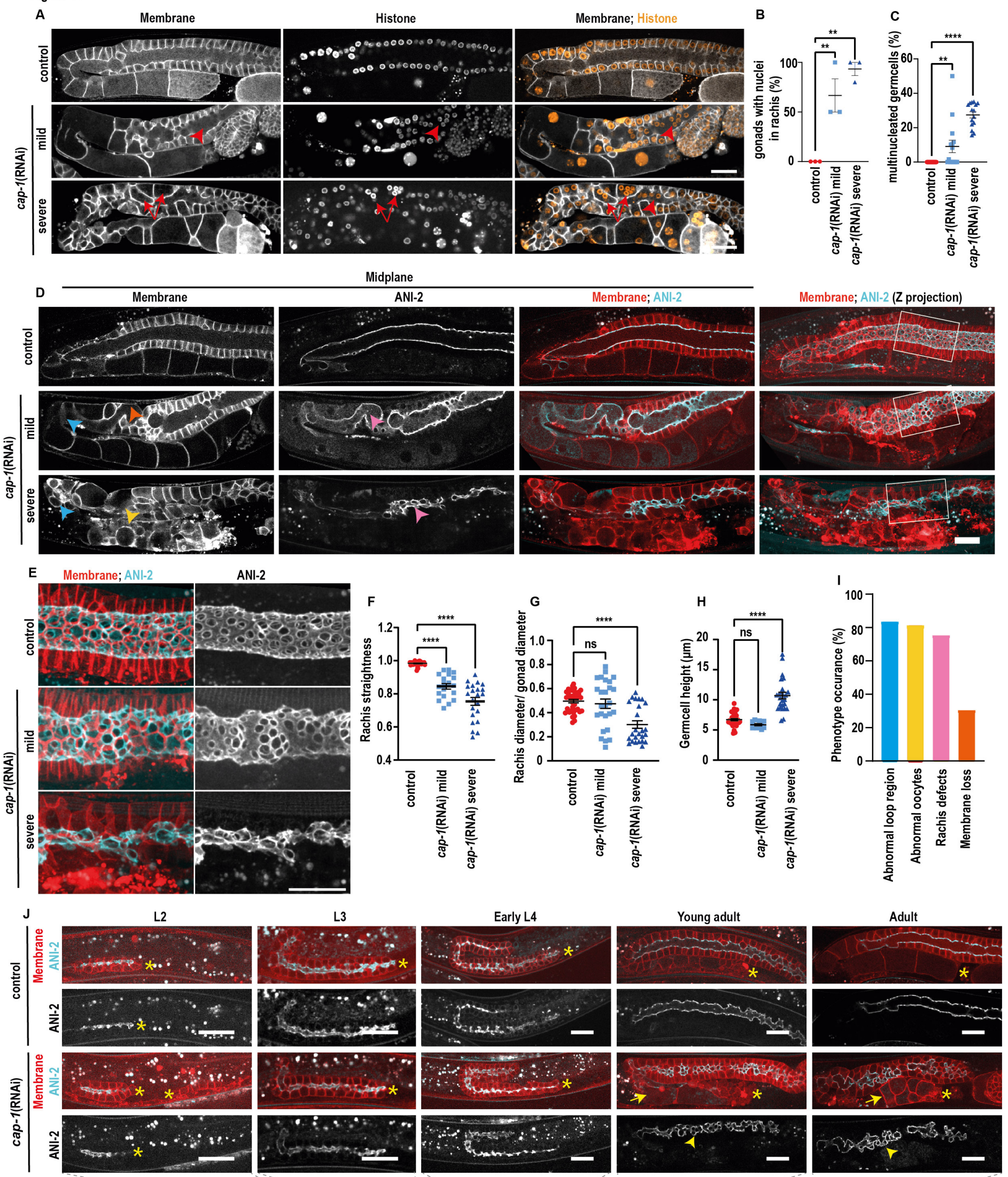

$\left.\underbrace{0 \mathrm{hr}}_{\substack{\text { embryo } \\ \text { laid }}} \substack{\text { embryo } \\ \text { hatches }}_{\text {Larval stage-1 }}^{12 \mathrm{hrs}}{ }_{\text {Larval stage-2 }}^{27 \mathrm{hrs}}\right|_{\text {Larval stage-3 }} ^{36 \mathrm{hrs}}$ 
Figure - 3
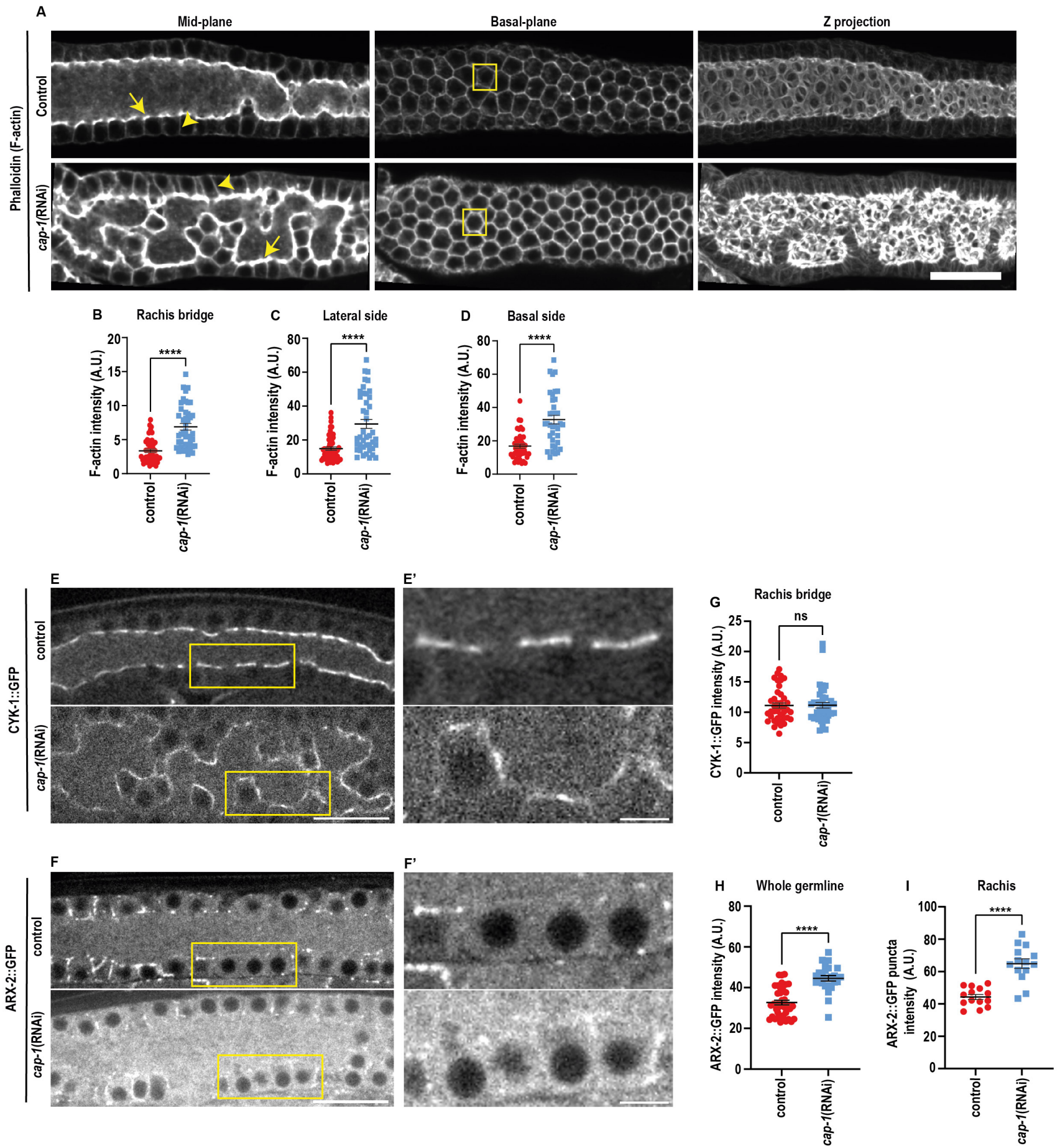
Figure -4
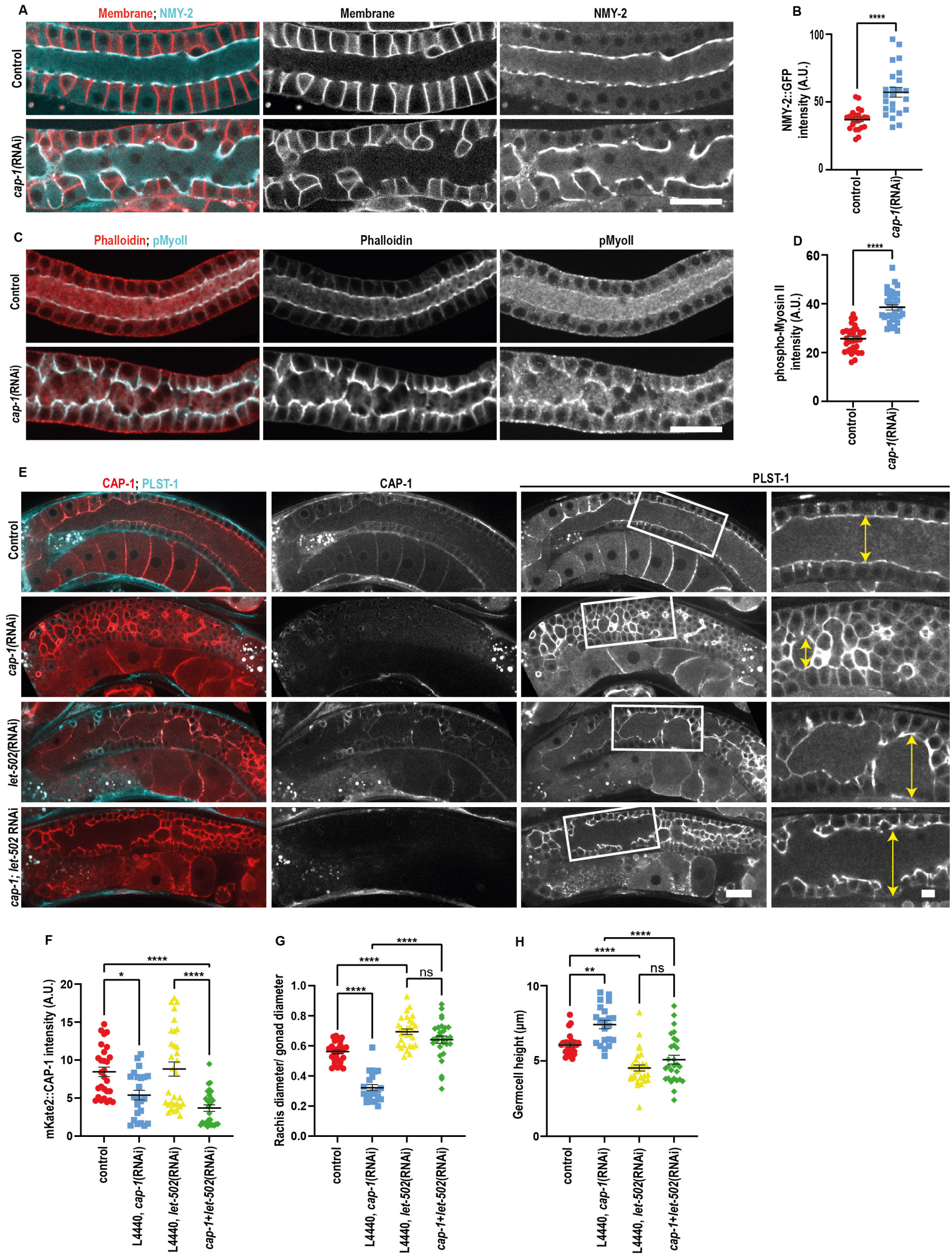
bioRxiv preprint doi: https://doi.org/10.1101/2021.10.03.462551; this version posted October 3, 2021. The copyright holder for this preprint (which was not certified by peer review) is the author/funder. All rights reserved. No reuse allowed without permission. 